\title{
Detection of the Faraday Chiral Anisotropy
}

Caridad, José M.; Tserkezis, Christos; Santos, Jaime E.; Plochocka, Paulina; Venkatesan, Munuswamy; Coey, J. M.D.; Mortensen, N. Asger; Rikken, Geert L.J.A.; Krsti, Vojislav

Published in:

Physical Review Letters

Link to article, DOI:

10.1103/PhysRevLett.126.177401

Publication date:

2021

Document Version

Publisher's PDF, also known as Version of record

Link back to DTU Orbit

Citation (APA):

Caridad, J. M., Tserkezis, C., Santos, J. E., Plochocka, P., Venkatesan, M., Coey, J. M. D., Mortensen, N. A., Rikken, G. L. J. A., \& Krsti, V. (2021). Detection of the Faraday Chiral Anisotropy. Physical Review Letters, 126(17), [177401]. https://doi.org/10.1103/PhysRevLett.126.177401

\section{General rights}

Copyright and moral rights for the publications made accessible in the public portal are retained by the authors and/or other copyright owners and it is a condition of accessing publications that users recognise and abide by the legal requirements associated with these rights.

- Users may download and print one copy of any publication from the public portal for the purpose of private study or research.

- You may not further distribute the material or use it for any profit-making activity or commercial gain

- You may freely distribute the URL identifying the publication in the public portal 


\title{
Detection of the Faraday Chiral Anisotropy
}

\author{
José M. Caridad, ${ }^{1,2,3}$ Christos Tserkezis, ${ }^{4}$ Jaime E. Santos $\odot,{ }^{5,6}$ Paulina Plochocka, ${ }^{7,8}$ Munuswamy Venkatesan, ${ }^{1}$ \\ J. M. D. Coey, ${ }^{1}$ N. Asger Mortensen $\odot,{ }^{3,4,9}$ Geert L. J. A. Rikken, ${ }^{7}$ and Vojislav Krstić $\circledast^{1,10, *}$ \\ ${ }^{1}$ School of Physics, CRANN, Amber Research Centre, Trinity College Dublin, College Green, Dublin 2, Ireland \\ ${ }^{2}$ DTU Physics, Technical University of Denmark, 2800 Kongens Lyngby, Denmark \\ ${ }^{3}$ Center for Nanostructured Graphene, Technical University of Denmark, 2800 Kongens Lyngby, Denmark \\ ${ }^{4}$ Center for Nano Optics, University of Southern Denmark, Campusvej 55 DK-5230 Odense, Denmark \\ ${ }^{5}$ Centro de Física, Universidade do Minho, P-4710-057 Braga, Portugal \\ ${ }^{6}$ Instituto de Polímeros e Compósitos, Universidade do Minho, P-4800-058 Guimarães, Portugal \\ ${ }^{7}$ Laboratoire National des Champs Magnétiques Intenses, UPR3228, CNRS/INSA/UGA/UPS, Toulouse and Grenoble, France \\ ${ }^{8}$ Department of Experimental Physics, Faculty of Fundamental Problems of Technology, \\ Wroclaw University of Science and Technology, Wroclaw, Poland \\ ${ }^{9}$ Danish Institute for Advanced Study, University of Southern Denmark, Campusvej 55 DK-5230 Odense, Denmark \\ ${ }^{10}$ Department of Physics, Friedrich-Alexander-University Erlangen-Nürnberg (FAU), Staudtstr. 7, 91058 Erlangen, Germany
}

(Received 29 July 2020; accepted 23 February 2021; published 27 April 2021)

\begin{abstract}
The connection between chirality and electromagnetism has attracted much attention through the recent history of science, allowing the discovery of crucial nonreciprocal optical phenomena within the context of fundamental interactions between matter and light. A major phenomenon within this family is the so-called Faraday chiral anisotropy, the long-predicted but yet unobserved effect which arises due to the correlated coaction of both natural and magnetically induced optical activities at concurring wavelengths in chiral systems. Here, we report on the detection of the elusive anisotropic Faraday chiral phenomenon and demonstrate its enantioselectivity. The existence of this fundamental effect reveals the accomplishment of envisioned nonreciprocal electromagnetic metamaterials referred to as Faraday chiral media, systems where novel electromagnetic phenomena such as negative refraction of light at tunable wavelengths or even negative reflection can be realized. From a more comprehensive perspective, our findings have profound implications for the general understanding of parity-violating photon-particle interactions in magnetized media.
\end{abstract}

DOI: 10.1103/PhysRevLett.126.177401

The interplay between electromagnetism and chirality possesses a rich and long history, comprising cornerstone discoveries that have largely contributed to our understanding of the electromagnetic properties of matter and the wave nature of light [1-3]. The exploration of this interdisciplinary field was initiated during the 19th century, when Arago discovered the natural optical activity [(NOA), quantified by a parameter $\alpha$ ], an enantioselective, nonlocal response in media that lacks mirror symmetry (chiral systems). Later, Faraday detected the magnetically induced optical activity [(MOA), quantified by a parameter $\beta]$, an omnipresent phenomenon resulting from the breaking of the time-reversal symmetry by a magnetic field $\vec{B}$ perpendicular to the optical polarization [1-3]. Despite their different physical roots, NOA and MOA are both manifested in a different absorption or emission (dichroism) or refraction (birefringence) between left and right circularly polarized light. Such phenomenological resemblances inspired several scientists, including Pasteur, to search for possible links between these two independent phenomena [3-22]. The most straightforward magnetooptical (MO) phenomenon of this kind would emerge as a direct correlated coaction of NOA and MOA and would exhibit a unique rotation of polarization simultaneously displaying both nonreciprocity and enantioselectivity. As such, this phenomenon breaks both time-reversal and parity symmetry and results in optical anisotropies appearing in chiral systems with opposite sign for left-handed (LH) and right-handed $(\mathrm{RH})$ enantiomers [Fig. 1(a)] [7-9,20,21]. Hereafter, we refer to the nonreciprocal and enantioselective (chiral) MO response occurring due to the simultaneous presence of both NOA and MOA at coinciding wavelengths as Faraday chiral anisotropy (FCA). We emphasize the diverse physical origin of the FCA with respect to other chiral MO effects such as the so-called [5,6] and already observed [9-16] magneto-chiral anisotropy. The latter anisotropy phenomenon is not a correlated effect but only a magnetic modulation of the NOA in chiral media subject to an external $\vec{B}$ [4-10]. Material systems exhibiting the FCA are a new class of metamaterials predicted to give rise to a host of exotic behaviors. These include negative refraction [23-26] over broad frequency ranges, controlled by an external $\vec{B}[8,20,21]$, and negative reflection, an effect where both reflected and incident light beams are on the 


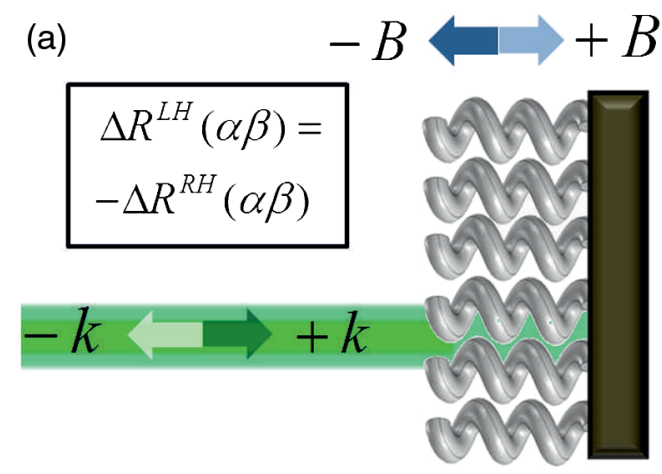

(b)

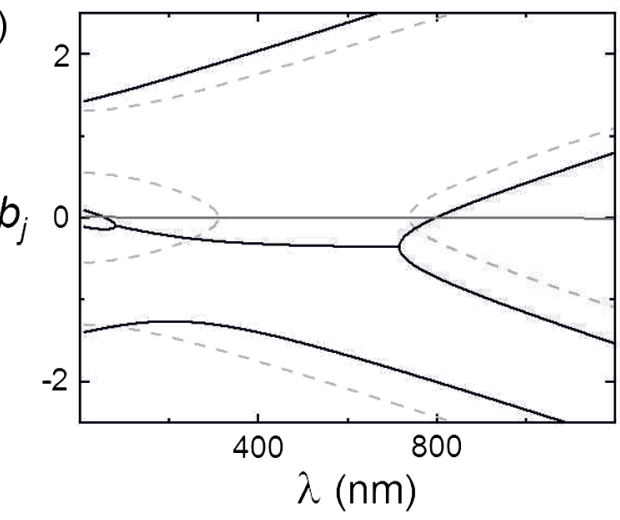

FIG. 1. (a) Arrays of ferromagnetic nanohelices are subjected to the external magnetic field $( \pm B)$, collinear to their helical axis (blue arrows). Samples are excited (dark arrow) with linearly polarized light $( \pm k)$, which propagates parallel to the helical axis, too. The normal reflectance (white arrow) difference $\Delta R=$ $R(+B)-R(-B)$ does not vanish due to the FCA $(\alpha \beta)$ and has opposite sign for LH and RH enantiomers. (b) Real part of the solutions $b_{j}$ of the dispersion relation Eq. (2) in the absence (dashed) and presence (continuous) of MOA $\beta B$. The FCA drives some polarization modes to exhibit opposite group and phase velocities at tunable wavelengths (see wavelengths slightly below $800 \mathrm{~nm}$ in this panel).

same side of the normal plane to the reflecting surface [19]. Furthermore, the FCA effect is of a more general nature and arises in any system simultaneously exhibiting both parity violation and Faraday rotation [7,9]. Therefore, the FCA is also expected to occur in systems composed of parityviolating, photon-particle interactions in a magnetic environment, including axion electrodynamics in magnetized plasma [27] or the electromagnetic response of chiral Weyl fermions in semimetals [28] exposed to an external $\vec{B}$. Nonetheless, no directly correlated coaction of NOA and MOA has been observed up to date, despite some recent revealing progress achieved at a theoretical level from different viewpoints. Eritsyan and Gevorgyan [7,8] envisioned and described formally the FCA in helical media within a linear expansion of the dielectric constant in terms of the wave vector of light $\vec{k}$ and $\vec{B}$. Other groups independently predicted the existence of such curious MO phenomenon, too: Engheta and Jaggard, who referred to such linear, nonreciprocal systems displaying NOA and MOA as Faraday chiral [17]; and Rikken and Raupach [9], who coined it cascaded MO effect due to simultaneous action of both NOA and MOA. Intriguingly, despite being broadly predicted [7-9,17-22] and sought after [9,14], the FCA is still unobserved. Specifically, since MOA is a ubiquitous effect, any medium displaying NOA (i.e., any chiral system) should exhibit the Faraday chiral phenomenon, too. This oddity can be resolved emphasizing that selected chiral systems with large NOA and MOA responses at coincident wavelengths are required to detect this effect. In particular, the MO response of biaxial chiral systems such as artificial ferromagnetic helical nanostructures is expected to be governed by the FCA [7,8,20,21]. All such media, including different types of structures like ferromagnetic nanohelices [21], cholesteric liquid crystals [8], or garnet particles arranged in helical shapes [22], are characterized by the same dielectric tensor which represents the simplest and most general description of periodically inhomogeneous media $[8,21]$. For our case [see Fig. 1(a)] of colinear $\vec{B}, \vec{k}$, and principal axis ( $z$ direction) this one reads

$$
\epsilon(z)=\epsilon_{m} \cdot\left(\begin{array}{ccc}
1+\delta_{\epsilon} \cos (2 a z) & \delta_{\epsilon} \sin (2 a z)+\frac{i \beta B}{\epsilon_{m}} & 0 \\
\delta_{\epsilon} \sin (2 a z)-\frac{i \beta B}{\epsilon_{m}} & 1-\delta_{\epsilon} \cos (2 a z) & 0 \\
0 & 0 & 1-\delta_{\epsilon}
\end{array}\right)
$$

with $\epsilon_{m}=\left(\epsilon_{1}+\epsilon_{2}\right) / 2, \delta_{\epsilon}=\left(\epsilon_{1}-\epsilon_{2}\right) /\left(\epsilon_{1}+\epsilon_{2}\right), \epsilon_{1}, \epsilon_{2}$ are the principal axis of $\epsilon(z)$ in a coordinate frame $\left(x^{\prime}, y^{\prime}, z\right)$ (local to the structure) that rotates with respect to the Cartesian laboratory coordinate frame $(x, y, z)$. We remark that this dielectric tensor contains the two key elements needed for the FCA to occur in the system, MOA and NOA. In other words, the dielectric tensor does not contain any FCA term explicitly since this effect arises from the generic coupling of NOA and MOA in the system. MOA is reflected in the complex, off-diagonal components $\beta|\vec{B}|=\beta B$ of the anisotropic tensor $(\vec{g} \equiv \beta \vec{B}$ is also called gyration vector $[8,21])$. NOA is here caused by the twisting of the medium and is reflected in the parameter $\alpha=c a /\left(\omega \sqrt{\epsilon_{m}}\right)=\lambda /\left(p_{0} \sqrt{\epsilon_{m}}\right)$, where $a=2 \pi / p_{0}, p_{0}$ is the pitch of the helix, $c$ is the speed of light and $\omega, \lambda$ are the angular frequency and wavelength of the incident wave, respectively. Light propagation in these media at normal incidence is represented in terms of its two circular components [7,8,20,21]. After substitution of such fields in the homogeneous Maxwell equations that describe the problem, with the dielectric tensor given by Eq. (1), one finds that the corresponding dispersion relation is given by the quartic expression $[8,21]$

$$
b_{j}^{4}+a_{1} b_{j}^{2}+a_{2} b_{j}+a_{3}=0
$$


where the roots $b_{j}(j=1,2,3,4)$ are the dimensionless form of wave vector $k_{j}=b_{j} \omega n_{m} / c$. Here, $n_{m}$ is the refraction index of the medium, $a_{1}=-2\left(1+\alpha^{2}\right)$, $a_{2}=4 \alpha \beta B / \epsilon_{m}$, and $a_{3}=-2 \alpha^{2}+\left(1-\delta_{\epsilon}\right)-\beta B / \epsilon_{m}$. We highlight that the FCA here stems from the unconventional odd-powered term $a_{2}$ containing the product $\alpha \beta$, that is, the coupling of NOA and MOA. To illustrate this, we note that if $a_{2}=0$ (for instance, if $\beta=0$ ), the dispersion relation is reciprocal (biquadratic). Roots are in this case $b_{j} \equiv b_{j}^{0}$, those of a conventional (one-dimensional) periodic helical medium [Fig. 1(b), dashed lines] [20], symmetric with respect to the axis $b_{j}=0$. Two out of the four modes develop a gap (origin of NOA).

The situation changes dramatically if $a_{2} \neq 0$ (i.e., $\alpha \beta \neq 0$ ). The dispersion relation is nonreciprocal in this case [see Fig. 1(b) continuous lines], and the four roots read $b_{1,2} \approx b_{1,2}^{0}+\omega c^{-1}(\alpha \beta B \zeta)$ and $b_{3,4} \approx b_{3,4}^{0}-\omega c^{-1}(\alpha \beta B \zeta)$ [7], where the parameter $\zeta=\left[\left(\omega \delta_{\epsilon} \epsilon_{m} / c\right)^{2}+4 \alpha^{2} \epsilon_{m}\right]^{-1 / 2}$. These four solutions are nonsymmetric with respect to $b_{j}=0$ due to the simultaneous action of NOA and MOA. Importantly, this implies that $\vec{k}$ has a different magnitude for forward and backward propagation, a key signature of Faraday chiral media (cf. also Refs. [7,8,21]). Only at one point within the NOA resonance, light propagation is reciprocal, where $\vec{k}$ is identical in magnitude for forward and backward propagation. This leads to zero crossings in the transmission and reflection anisotropies (as shown below). The four modes $b_{j}$ make the polarization ellipses to experience different rotations depending on $\vec{k}$ and $\vec{B}$ directions. However, contrary to the cases of NOA or MOA alone, their simultaneous presence makes the magnitude of the rotation difference $\Delta \phi$ (per unit length) between the Faraday angles for forward and backward light propagation (with respect to $\vec{B}$ ) not to vanish in Faraday chiral media. Instead, $\Delta \phi=4(\omega / c) \alpha \beta B \zeta \neq 0($ as $\alpha \beta \neq 0)$ and manifests as nonreciprocal and enantioselective birefringence in refraction or dichroism in absorption and emission (real or imaginary part of $\Delta \phi$, respectively) [7].

The presence of the FCA in helical systems is thus envisioned to exhibit distinct scattering anisotropy and enantioselective behavior. Normal reflection is particularly suitable to detect the FCA since the MO response in this scattering configuration is exclusively due to the term $\alpha \beta$ (other chiral MO effects vanish) [21]. As such, due to the term $a_{2}$ in Eq. (2), the calculated reflectance difference $\Delta R=R(+B)-R(-B)$ as a function of $\lambda$ is, in general, nonzero (the system is non-reciprocal except at one point within the NOA resonance). It also shows a sign reversal for the two enantiomers of the system (RH and LH helices). In other words, $\Delta R^{\mathrm{LH}}(\alpha \beta)=-\Delta R^{\mathrm{RH}}(\alpha \beta) \neq 0$ [see Figs. 1(a), 3 and 4]. We further emphasize that none of these distinct Faraday chiral features are present in helical systems if $\beta=0$, regardless of the presence or not of any other chiral MO effect (see Supplemental Material [29]). (a)

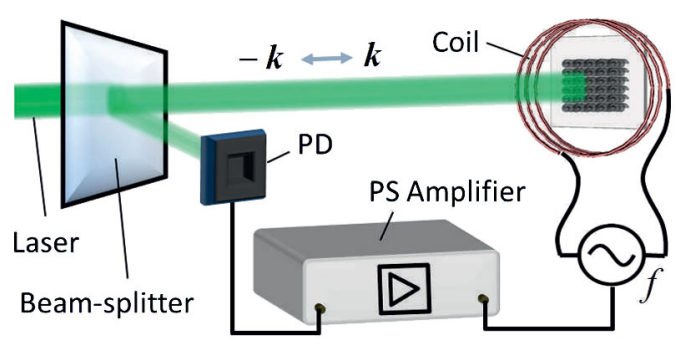

(b)

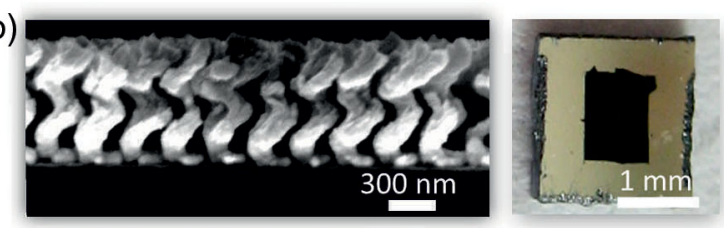

FIG. 2. (a) Schematic of the experimental setup. The external $\vec{B}$ is generated by a coil and is collinear with the light propagation direction and the helical axis. Samples are excited with (linearly polarized) laser wavelengths 440, 540, 633, and $741 \mathrm{~nm}$. The optical reflectance is detected by a silicon photodiode (PD). The intensity difference $(\Delta R)$ between the two magnetic field directions is phase-sensitively (PS) detected by a lock-in amplifier at the frequency $f=19 \mathrm{~Hz}$ of the alternating magnetic field. (b) (Right panel) optical image showing the array of ferromagnetic (nickel) nanohelices on top of an $80 \mathrm{~nm}$ thick silver film. (Left panel) scanning electron micrograph of one of the enantiomers, showing well-defined nickel nanohelices with a pitch of $\sim 320 \mathrm{~nm}$.

By taking into account these premises, we demonstrate here the unequivocal detection of the missing FCA in the normally reflected $\mathrm{MO}$ signal of nickel helical nanostructures [Figs. 1(a),2(a)]. As aforementioned, NOA in artificial helical media is exclusively caused by the twisting of the medium $[7,8,21]$. Also, the simultaneously enhanced MOA in these nanostructures is primarily due to the use of a ferromagnetic material [21]. The combination of these two features allows us to achieve enhanced NOA and MOA at concurring wavelengths, hence to detect the FCA. Figure 2(a) depicts the specific experimental setup [29] used here to measure the FCA in ferromagnetic nanohelices in reflection. We use light from a laser source as the incident radiation and, to increase sensitivity, the magnetic field is alternated in the two opposite configurations $( \pm B)$ so the reflectance difference $\Delta R=R(+B)-R(-B)$ is measured with a phase-sensitive detection method. Factors related to sample geometry are eliminated by dividing $\Delta R$ by the total, static reflectance signal $R(0)$. Therefore, $\Delta R / R(0)$ normalized by $B$ corresponds to the Faraday chiral MO factor $\eta$ :

$$
\eta=\frac{\Delta R}{R(0)} B^{-1}
$$

We have fabricated [29-34] regular arrays of artificial nickel nanohelices with $\sim 1$ turn and a pitch of $\sim 320 \mathrm{~nm}$ 

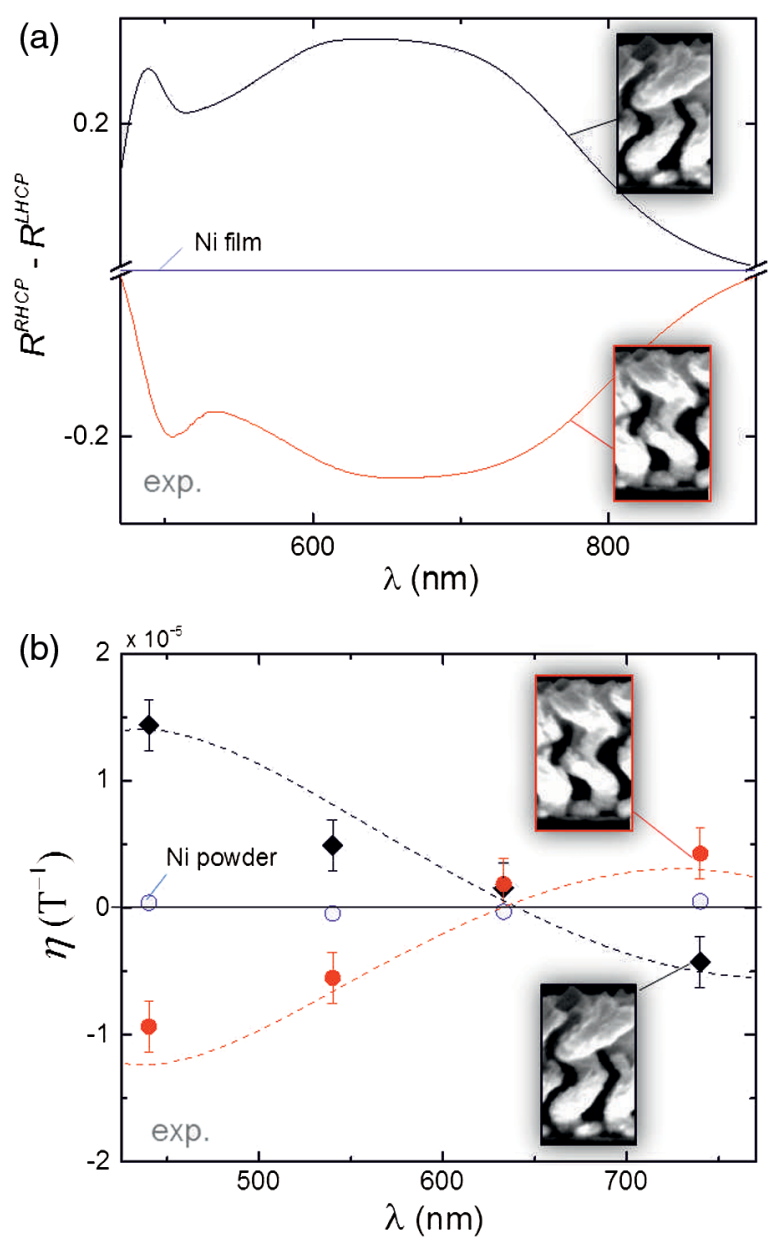

FIG. 3. (a) NOA of our samples measured as the normal reflectance difference $R^{\mathrm{RHCP}}-R^{\mathrm{LHCP}}$ between $\mathrm{RH}$ and $\mathrm{LH}$ circular polarized light in RH (black) and LH (red) helices. Both enantiomers show a clear maximum of $\sim 0.3$ within a region around $\sim 640 \mathrm{~nm}$. A zero reflectance difference is measured for a nickel thin film (blue). (b) MO signal in reflection $\eta$ for both $\mathrm{RH}$ (black) and LH (red) helices plotted as a function of $\lambda$, exhibiting zero-crossing close to the NOA maximum and a change of sign at both sides of this position in each enantiomer. Dashed lines are guide the eye. For reference, an $\eta$ below $5 \times 10^{-7} \mathrm{~T}^{-1}$ was measured for nickel powder (blue circles), indicating our setup detection limit. Measurements were performed at the external magnetic field strength $B=0.375 \mathrm{~T}$.

[Fig. 2(b)] in both types of enantiomer- $\mathrm{RH}$ and $\mathrm{LH}$ helices-as our specific ferromagnetic chiral medium.

Figure 3(a) shows the measured reflectance difference at zero $\vec{B}$ between right- and left-handed circular polarized light, $R^{\mathrm{RHCP}}-R^{\mathrm{LHCP}}$ in both types of helices. This signal represents the NOA of the system and exhibits a relatively broad response with a maximum of magnitude $(\sim 0.3)$ at a wavelength $\lambda^{\max } \sim 640 \mathrm{~nm}$ for both enantiomers.

Figure 3(b) shows the experimental $\eta(\lambda)$ plotted for the two enantiomers under study in the wavelength range 440 to $740 \mathrm{~nm}$ and at $B=0.375$ T. $\eta(\lambda)$ shows opposite behavior in both RH and LH enantiomers with similar magnitudes.
Also, its magnitude increases when increasing $B$ [29]. Strikingly, we observe a zero crossing of $\eta(\lambda)$ occurring at $\sim 633 \mathrm{~nm}$, positioned very close to $\lambda^{\max }$ (NOA maximum) and with a nonzero MOA [29]. Moreover, $\eta(\lambda)$ changes sign at the two opposite sides of $\lambda^{\max }$ in both enantiomers.

As aforementioned, the latter two findings are the unique hallmarks of the predicted FCA [7,8,21] and thus demonstrate its detection. The magnitude of the anisotropy factor $\eta$ is $\sim 10^{-5} \mathrm{~T}^{-1}$, which is in good agreement to the common assumption $[9,14,16]$ given by the product of NOA and MOA (independently measured) in our samples: $\alpha \sim 0.3$ [Fig. 2(a)] and $\beta \sim 10^{-4} \mathrm{~T}^{-1}$, respectively [29].
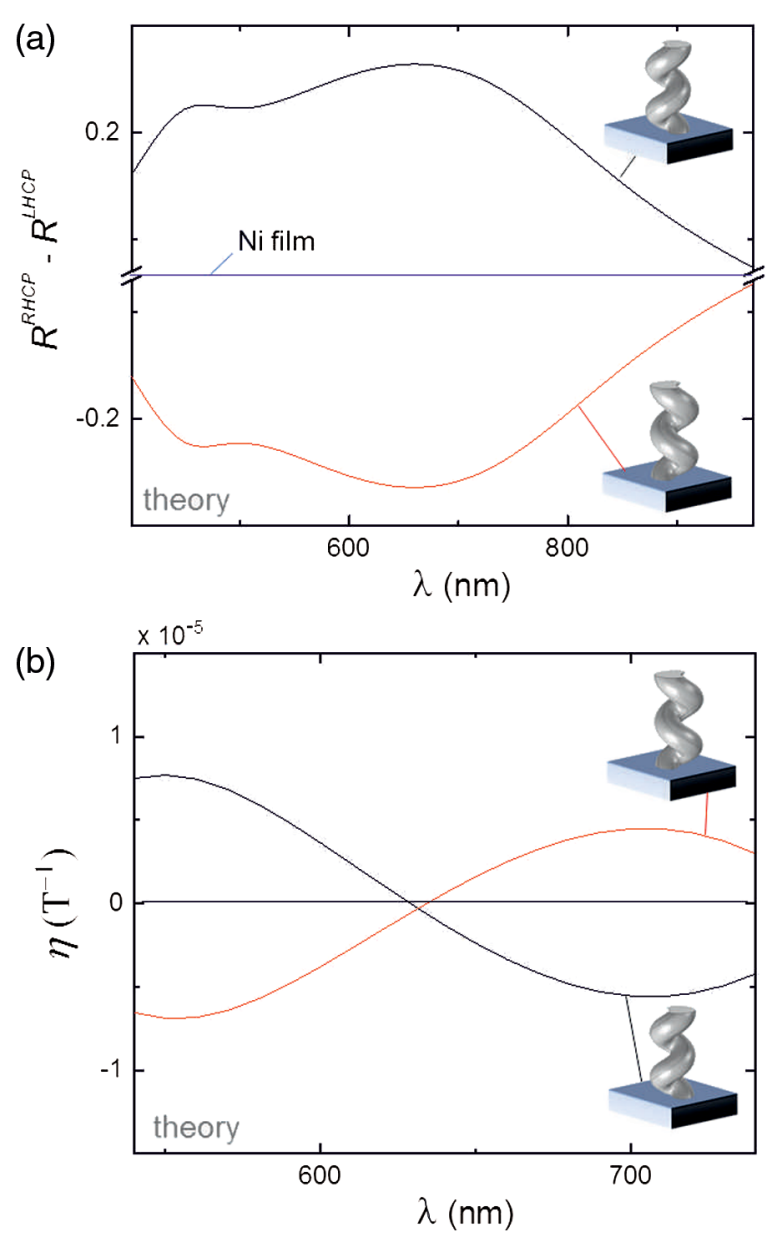

FIG. 4. (a) NOA. Calculated reflectance difference between right- $\left(R^{\mathrm{RHCP}}\right)$ and left- $\left(R^{\mathrm{LHCP}}\right)$ handed circular polarized light in $\mathrm{RH}$ (black) and LH (red) helices and a nickel film (blue). The NOA maximum appears within the range of 550 to $770 \mathrm{~nm}$. (b) Calculated MO signal $\eta$ in normal reflection of both $\mathrm{RH}$ (black) and LH (red) helices plotted as a function of $\lambda$, exhibiting a clear Faraday chiral MO behavior (zero crossing close to NOA maximum and corresponding sign change for both enantiomers). Calculations were undertaken with linearly polarized light (as in the experiment). We note that the exact NOA maximum in (a) and zero crossing in (b) depend slightly on the separation distance between individual helices (see Ref. [29]). 
We additionally corroborate the observation of the FCA by undertaking advanced electromagnetic simulations [29], calculating the expected MO response of our nickel nanohelices in normal reflection. In these calculations, NOA is implicitly considered by the helical shape of the structures and MOA is included in the system by accounting for the permittivity tensor of the ferromagnetic material (nickel) in the presence of $\vec{B}$ [29]. Figure 4(a) shows the calculated reflectance difference at zero $\vec{B}$, $R^{\mathrm{RHCP}}-R^{\mathrm{LHCP}}$, exhibiting a broad response $[29,35]$ with a maximum $(\sim 0.3)$ at $\lambda^{\max } \sim 665 \mathrm{~nm}$ for both enantiomers. Thus, these features as well as the overall line shape are in good agreement with our NOA response measured [Fig. 2(a)]. Figure 4(b) depicts the calculated $\eta(\lambda)$ for the two enantiomers of the chiral system under study. $\eta(\lambda)$ exhibits a zero crossing occurring at the NOA resonance $(\sim 650 \pm 25 \mathrm{~nm})$ and with opposite sign of the slope for each enantiomer. The magnitude found for $\eta(\lambda)$ at these wavelengths is $\sim 10^{-5} \mathrm{~T}^{-1}$. Remarkably, both line shape and magnitude of the calculated $\eta(\lambda)$ are in excellent quantitative agreement with our experiments [Fig. 2(b)], especially when considering that no theory has been able to model successfully any chiral MO effect up to date [9-16]. Additional calculation details can be found in the Supplemental Material [29].

In conclusion, we have reported the observation of the long-missing Faraday chiral anisotropy, the anisotropic optical response occurring in any system with simultaneous optical activity (NOA) and Faraday rotation (MOA). The existence of this nonreciprocal and enantioselective MO effect reveals the experimental realization of the envisioned Faraday chiral media [7,8,17-21]. Our work opens the door to the exploration of a rich number of exotic wave properties predicted to occur in Faraday chiral media [17-22] such as negative reflection [19], as well as the realization of predicted nonreciprocal electromagnetic devices, as, for example, wavelength-selective on-chip optical isolators $[21,36]$ for photonic circuitry. In a more general context, the FCA has profound implications for the understanding of parity-violating photon-particle interactions. Such situations can be found in photon-axion oscillations in a magnetized plasma [27] and the electromagnetic response of chiral Weyl fermions in semimetals [28] in an external magnetic field.

The authors thank A. Gevorgyan for useful discussions and the Advanced Microscopy Laboratory, CRANN, TCD for assistance during sample preparation. This work was supported by the following grants: Science Foundation Ireland PI-Grant 08/IN.1/I1873; CSET 08/CE/I1432; European Union EuroMagNET II Programme; Cycle 4 of the Programme for Research in Third-Level Institutions (PRTLI4) Ireland; Programme Investissements d'Avenir under the program ANR-11-IDEX-0002-02, ref. ANR-10LABX-0037-NEXT; Vinnova (Project ID: 2019-02878); the MINECO Ramón y Cajal program (No. RYC2019-
028443-I); the Center for Nanostructured Graphene (Danish National Research Foundation, Project No. DNRF103); VILLUM FONDEN (Grant No. 16498); 2020 funding and HPC Centre at SDU; the Operational Competitivness and Internationalization Programme, COMPETE 2020 (Project No. POCI-01-0247-FEDER39479); the Portugese Foundation for Science and Technology, FCT (Project No. FCT-UID/FIS/04650/ 2020) and the Deutsche Forschungsgemeinschaft (DFG, German Research Foundation) - Project-ID 429529648 TRR 306 QuCoLiMa ("Quantum Cooperativity of Light and Matter").

*Corresponding author. vojislav.krstic@fau.de

[1] L. D. Barron, Molecular Light Scattering and Optical Activity (Cambridge University Press, Cambridge, England, 2004).

[2] I. V. Lindell, A. H. Sihvola, S. A. Tretyakov, and A. J. Viitanen, Electromagnetic Waves in Chiral and Bi-Isotropic Media (Artech House Inc., Boston, 1994).

[3] G. H. Wagnière, On Chirality and the Universal Asymmetry: Reflections on Image and Mirror Image (John Wiley \& Sons, New York, 2008).

[4] N. B. Baranova and B. Y. Zeldovich, Mol. Phys. 38, 1085 (1979).

[5] G. H. Wagnière and A. Meier, Chem. Phys. Lett. 93, 78 (1982).

[6] L. D. Barron and J. Vrbancich, Molec. Phys. 51, 715 (1984).

[7] O. S. Eritsyan, Mol. Cryst. Liq. Cryst. 348, 79 (2000).

[8] A. H. Gevorgyan, Mol. Cryst. Liq. Cryst. 382, 1 (2002).

[9] G. L. J. A. Rikken and E. Raupach, Phys. Rev. E 58, 5081 (1998).

[10] G. J. L. A. Rikken and E. Raupach, Nature (London) 390, 493 (1997).

[11] C. Train, R. Gheorghe, V. Krstić, L. M. Chamoreau, N. S. Ovanesyan, G. J. L. A. Rikken, M. Gruselle, and M. Verdaguer, Nat. Mater. 7, 729 (2008).

[12] R. Sessoli, M.-E. Boulon, A. Caneschi, M. Mannini, L. Poggini, F. Wilhelm, and A. Rogalev, Nat. Phys. 11, 69 (2015).

[13] E. Raupach, G. L. J. A. Rikken, C. Train, and B. Malzieux, Chem. Phys. 261, 373 (2000).

[14] C. Koerdt, G. Düchs, and G. L. J. A. Rikken, Phys. Rev. Lett. 91, 073902 (2003).

[15] P. Kleindienst and G. H. Wagnière, Chem. Phys. Lett. 288, 89 (1998).

[16] S. Eslami, J. G. Gibbs, Y. Rechkemmer, J. V. Slageren, M. Alarcón-Correa, T.-C. Lee, A. G. Mark, G. L. J. A. Rikken, and P. Fischer, ACS Photonics 1, 1231 (2014).

[17] N. Engheta and D. L. Jaggard, IEEE Trans. Antennas Propag. 40, 367 (1992).

[18] W. S. Weiglhofer and A. Lakhtakia, Microwave Opt. Technol. Lett. 17, 405 (1998).

[19] T. G. Mackay and A. Lakhtakia, Microwave Opt. Technol. Lett. 50, 1368 (2008). 
[20] I. Bita and E. L. Thomas, J. Opt. Soc. Am. B 22, 1199 (2005).

[21] A. H. Gevorgyan and G. A. Vardanyan, Proc. SPIE 5218, Complex Mediums IV: Beyond Linear Isotropic Dielectrics (2003), https://doi.org/10.1117/12.507791.

[22] A. Christofi and N. Stefanou, Opt. Lett. 38, 4629 (2013).

[23] V. G. Veselago, Sov. Phys. Usp. 10, 509 (1968).

[24] C.-W. Qiu, L.-W. Li, H.-Y. Yao, and S. Zouhdi, Phys. Rev. B 74, 115110 (2006).

[25] R. A. Shelby, D. R. Smith, and S. Schultz, Science 292, 77 (2001).

[26] D. R. Smith, S. Schultz, N. Kroll, and R. A. Shelby, Left Handed Composite Media, US Patent No. US6791432B2 (2000).

[27] A. K. Ganguly, P. Jain, and S. Mandal, Phys. Rev. D 79, 115014 (2009).

[28] M. Kargarian, M. Randeria, and N. Trivedi, Sci. Rep. 5, 12683 (2015).
[29] See Supplement Material at http://link.aps.org/supplemental/ 10.1103/PhysRevLett.126.177401 for a description of the experimental and theoretical methods, as well as additional experimental data and calculations.

[30] J. M. Caridad, D. McCloskey, J. F. Donegan, and V. Krstić, Appl. Phys. Lett. 105, 233114 (2014).

[31] J. M. Caridad, D. McCloskey, F. Rossella, V. Bellani, J. F. Donegan, and V. Krstić, ACS Photonics 2, 675 (2015).

[32] J. M. Caridad, S. Winters, D. McCloskey, G. S. Duesberg, J. F. Donegan, and V. Krstić, Sci. Rep. 7, 45548 (2017).

[33] J. J. Gough, D. McCloskey, J. M. Caridad, V. Krstić, M. Müller, N. Giaponik, and A. L. Bradley, https://doi.org/ 10.1109/MetaMaterials.2015.7342532 (2015).

[34] J. M. Caridad, S. Winters, D. McCloskey, G. S. Duesberg, J. F. Donegan, and V. Krstić, Nanotechnology 29, 325204 (2018).

[35] J. K. Gansel et al., Science 325, 1513 (2009).

[36] A. Lakhtaria and R. Messier, Sculptured Thin Films: Nanoengineered Morphology and Optics (SPIE, Washington, 2005). 